\title{
Problemas associados ao uso de plantas medicinais comercializadas no Mercadão de Madureira, município do Rio de Janeiro, Brasil
}

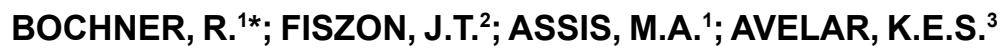

${ }^{1}$ Fiocruz/ICICT/LICTS, Avenida Brasil, 4365, Pavilhão Haity Mossatché, sala 206, CEP: 21045-900, Rio de JaneiroBrasil ${ }^{2}$ Fiocruz/ENSP/DCS, Rua Leopoldo Bulhões, 1480, sala 909, CEP: 21045-210, Rio de Janeiro-Brasil ${ }^{3}$ Fiocruz/IOC, Avenida Brasil, 4365, CEP: 21040-360, Rio de Janeiro-Brasil *rosany@icict.fiocruz.br

\begin{abstract}
RESUMO: O uso de plantas medicinais pela população brasileira é prática tradicional, sendo muitas vezes o único recurso utilizado na atenção básica de saúde. O uso terapêutico dessas plantas envolve várias etapas da cadeia produtiva, sendo a procedência, coleta, secagem, armazenamento, comércio, modo de preparo pelo usuário e uso. O objetivo desse trabalho documental, de caráter exploratório, foi levantar a produção científica existente sobre os problemas associados a cada uma dessas etapas e discutir as questões relacionadas à carência de estudos para comprovar a eficácia farmacológica e a ausência de riscos toxicológicos, bem como a prática de autodiagnóstico. As vinte plantas mais comercializadas em grande mercado do município do Rio de Janeiro em agosto de 2007 serviram de base para o levantamento documental do presente estudo. Dessas, seis apresentaram propriedades tóxicas comprovadas dependendo do preparo e uso, a arnica (Solidago chilensis Meyen), aroeira (Shinus terebinthifolius Raddi.), arruda (Ruta graveolens L.), babosa (Aloe vera L.), confrei (Symphytum officinale L.) e poejo (Mentha pulegium Lam. \& DC.). AAgência Nacional de Vigilância Sanitária aponta contra indicações para boldo-do-Chile (Peumus boldus Molina), chapéu-de-couro (Echinodorus macrophyllus Micheli), erva-cidreira (Lippia alba N.E.Br.), erva-de-bicho (Polygonum spp.), espinheira-santa (Maytenus spp.), picão (Bidens pilosa L.), poejo (Mentha pulegium Lam.) e tanchagem (Plantago major L.). O abajerú, arnica, boldo-do-Chile, confrei, erva-de-bicho e espinheira-santa tiveram relato de problemas de identificação na coleta e comercialização frente a outras morfologicamente semelhantes. Plantas cultivadas e silvestres apresentam variabilidade de princípios ativos influenciados por fatores ambientais e genéticos, como chapéu-de-couro (Echinodorus macrophyllus Micheli), erva-cidreira (Lippia alba N.E.Br.) e erva-de-bicho (Polygonum spp.). A contaminação e o comprometimento da preservação dos princípios ativos pela secagem e armazenamento inadequados foram relatados para o guaco (Mikania glomerata Sprengel), camomila (Chamomilla recutita L.), erva-cidreira, chapéu-de-couro e boldo-do-Chile (Peumus boldus Molina). Pode-se constatar que todas as etapas da cadeia produtiva das plantas medicinais apresentam desafios para que se possa garantir identificação da espécie, disponibilidade, qualidade, segurança e eficácia de uso.
\end{abstract}

Palavras-chave: medicina popular, toxicidade, cadeia produtiva das plantas medicinais

ABSTRACT: Problems associated with the use of medicinal plants commercialized in "Mercadão de Madureira", Rio de Janeiro City, Brazil. The use of medicinal plants by the Brazilian population is a traditional practice and is often the main resource used in primary healthcare. The therapeutic use of these plants involves several steps in the supply chain: origin, harvest, drying, storage, form of preparation by the user and use. The aim of this documental study of exploratory nature was to survey the scientific literature about the problems associated with each of those steps and discuss the issues related to the lack of studies to prove the pharmacological efficacy and the absence of toxicological risks, as well as the autodiagnosis practice. The 20 plants most commercialized in a large market of Rio de Janeiro City in August 2007 were the basis for the documental survey of the present study. Of these, six had proven toxic properties depending on their preparation and use: arnica (Solidago chilensis Meyen), aroeira (Shinus terebinthifolius Raddi.), rue (Ruta graveolens L.), "babosa" (Aloe vera L.), comfrey (Symphytum officinale L.) and pennyroyal (Mentha pulegium Lam. \& DC.). The National Agency

Recebido para publicação em 24/10/2010

Aceito para publicação em 21/05/2012

Rev. Bras. PI. Med., Botucatu, v.14, n.3, p.537-547, 2012. 
for Sanitary Surveillance shows contraindications for: "boldo-do-Chile" (Peumus boldus Molina), "chapéu-de-couro" (Echinodorus macrophyllus Micheli), lemon balm (Lippia alba N.E.Br.), "ervade-bicho" (Polygonum spp.), "espinheira-santa" (Maytenus spp.), "picão" (Bidens pilosa L.), pennyroyal (Mentha pulegium Lam.) and plantain (Plantago major L.). "Abajerú", arnica, "boldodo-Chile", comfrey, "erva-de-bicho" and "espinheira-santa" were reported to show identification problems in the harvest and in the commercialization compared to morphologically similar plants. Cultivated and wild plants showed variability in active principles influenced by environmental and genetic factors: "chapéu-de-couro" (Echinodorus macrophyllus Micheli), lemon balm (Lippia alba N.E.Br.) and "erva-de-bicho" (Polygonum spp.). Contamination and compromising of the preservation of active principles due to inadequate drying and storage was reported for guaco (Mikania glomerata Sprengel), camomile (Chamomilla recutita L.), lemon balm, "chapéu-de-couro" and "boldo-doChile" (Peumus boldus Molina). All stages of the supply chain of medicinal plants constitute challenges to ensure the proper species identification, availability, quality, safety, and efficacy of their use.

Key words: popular medicine, toxicity, supply chain of medicinal plants

\section{INTRODUÇÃO}

O uso de plantas medicinais pela população brasileira é uma prática tradicional (Ritter et al., 2002; Maioli-Azevedo \& Fonseca-Kruel, 2007), sendo muitas vezes o único recurso utilizado na atenção básica de saúde (Veiga Junior et al., 2005). A Organização Mundial de Saúde recomenda a difusão mundial dos conhecimentos necessários ao uso racional das plantas medicinais. Calixto (2000) aponta que os dados existentes até o fim do século XX ainda eram insuficientes para prover uma avaliação acurada da qualidade, eficácia e segurança da maior parte das plantas medicinais comercializadas mundialmente. No Brasil, um conjunto de resoluções e portarias delineia os instrumentos necessários à implantação da Política Nacional de Plantas Medicinais e Fitoterápicos, destacando-se a Relação Nacional de Plantas Medicinais de Interesse ao SUS (Brasil, 2009) e o Anexo I da Resolução da Diretoria Colegiada da ANVISA no 10 , que traz dados de nomenclatura, parte utilizada, posologia, modo de usar, via, indicações de uso, contraindicações e efeitos adversos, para 66 espécies de plantas medicinais utilizadas na preparação de drogas vegetais pela empresa fabricante (Anvisa, 2010a).

Embora haja preocupação explícita com a disseminação do uso de plantas medicinais pela população, ainda permanecem presentes as práticas tradicionais de dispensação das mesmas, que são adquiridas através do comércio popular.

Fabricantes e fornecedores de medicamentos homeopáticos e fitoterápicos devem atender aos critérios e especificações técnicas para a sua qualificação, estabelecidos na Resolução da Diretoria Colegiada da ANVISA no 14 (Anvisa, 2010b). Os mesmos procedimentos e cuidados ainda não estão desenvolvidos para a cadeia produtiva das plantas medicinais. A Política Nacional de Práticas Integrativas e Complementares no SUS aceita a premissa de que não deve ser subestimado o conhecimento popular sobre plantas medicinais. Afirma ainda que este só deva ser repassado depois da confirmação das propriedades atribuídas às plantas e do estabelecimento do uso seguro (Brasil, 2006). Não obstante, de acordo com Rodrigues \& Carlini (2002), menos de $0,5 \%$ da flora brasileira foi objeto de estudos fitoquímicos e farmacológicos.

O uso terapêutico dessas plantas envolve várias etapas que vão desde o cultivo até a administração. Embora essas plantas sejam popularmente consideradas terapêuticas, frequentemente possuem propriedades tóxicas desconhecidas pela população. Dados do Sistema Nacional de Informações Tóxico-FarmacológicasSINITOX (Sinitox, 2010) registram a ocorrência de 8.501 casos de intoxicação por plantas no Brasil no período de 2004 a 2008. Destas intoxicações, $12,4 \%$ estavam relacionadas a circunstâncias intencionais em que a vítima buscava propriedades farmacológicas da planta. No Rio de Janeiro foram observadas 415 intoxicações no mesmo período, sendo $12,8 \%$ relacionadas às mesmas circunstâncias intencionais.

O objetivo desse trabalho documental, de caráter exploratório, foi levantar a produção científica existente sobre os problemas associados às etapas da cadeia produtiva, sendo a procedência, coleta, secagem, armazenamento, comércio, modo de preparo pelo usuário e uso de plantas medicinais. Também discute problemas relacionados à carência de estudos para comprovar a eficácia farmacológica e a ausência de riscos toxicológicos, bem como a prática de autodiagnóstico. As vinte plantas mais comercializadas em grande mercado do município do Rio de Janeiro em agosto de 2007 serviram de base para o levantamento documental do presente estudo.

Rev. Bras. PI. Med., Botucatu, v.14, n.3, p.537-547, 2012. 


\section{MATERIAL E MÉTODO}

O Mercadão de Madureira foi selecionado para a realização de busca ativa das plantas mais comercializadas para o uso medicinal. Este mercado, que constitui o maior centro de distribuição de alimentos da Zona Norte do Município do Rio de Janeiro, atrai cerca de 80.000 pessoas por dia. A seleção das espécies das plantas para o estudo foi obtida em agosto de 2007, a partir de informações prestadas pelos 17 erveiros do Mercadão de Madureira, que comercializavam exclusivamente plantas medicinais e ritualísticas. Através da técnica de listagem livre, foi solicitado a cada erveiro que citasse pelo nome popular as espécies mais comercializadas, para fins medicinais, nos últimos sete dias. A compilação das informações obtidas permitiu a elaboração de listagem, das quais as 20 plantas citadas com maior frequência foram selecionadas para o estudo. As 20 plantas foram adquiridas na forma comum de comercialização, o que muitas vezes impedia a identificação botânica das espécies. Tal fato foi determinante na decisão de se utilizar exclusivamente fontes bibliográficas o que resultou em um trabalho documental, de caráter exploratório.

Para estabelecer a relação entre os nomes populares e científicos foram utilizados estudos que se concentraram nos mercados e feiras livres do município do Rio de Janeiro (Jacomassi \& Machado, 2003; Coulaud-Cunha et al., 2004; Azevedo \& Silva, 2006; Maioli-Azevedo \& Fonseca-Kruel, 2007; Mariot \& Barbieri, 2007; Arjona et al., 2007), complementados pela literatura consagrada que apresentava fotos, características, nomes científicos e populares das plantas selecionadas (Corrêa, 1984; Lorenzi \& Matos, 2002; Lameira \& Pinto, 2008; Saad et al., 2009).

Foi realizada uma pesquisa bibliográfica para identificar os estudos existentes a partir de duas buscas. Na primeira, foram selecionados todos os artigos que apresentavam nos títulos os nomes científicos de uma ou mais das 20 espécies contidas na listagem. Na segunda, foram selecionados os artigos que continham a palavra-chave "plantas medicinais". Para a busca foram utilizados os periódicos Revista Brasileira de Plantas Medicinais, Revista de Farmacognosia e Revista Brasileira de Biociências, abrangendo os períodos de 1998 a 2009, 1986 a 2009 e de 2004 a 2009, respectivamente.

Foi utilizada a definição de Fonte et al. (2004) para a cadeia produtiva de plantas medicinais como sendo a sequência que se estende desde o cultivo até a utilização pela população. Os artigos selecionados na pesquisa bibliográfica foram utilizados para identificar os problemas associados às seguintes etapas dessa cadeia produtiva: procedência, coleta, secagem, armazenamento, comércio, modo de preparo pelo usuário e uso. Os mesmos artigos também permitiram levantar problemas relacionados à carência de estudos para comprovar a eficácia farmacológica e a presença ou ausência de propriedades tóxicas nas 20 espécies. Ainda foi possível, com a mesma bibliografia, abordar aspectos da prática de autodiagnóstico no comércio de plantas medicinais.

A lista das plantas selecionadas foi comparada com o Anexo I da Resolução da Diretoria Colegiada da ANVISA no 10, de 9 de março de 2010 (Anvisa, 2010a) e com a Relação Nacional de Plantas Medicinais de Interesse ao SUS (Brasil, 2009).

\section{RESULTADO}

As 20 plantas selecionadas, segundo as informações prestadas pelos erveiros do Mercadão de Madureira, foram abajerú (Chrysobalanus icaco L. I Eugenia rotundifolia Casar.), arnica (Solidago chilensis Meyen), aroeira (Shinus terebinthifolius Raddi.), arruda (Ruta graveolens L.), babosa (Aloe vera L.), boldodo-Chile (Peumus boldus Molina), camomila (Chamomilla recutita L. (sin: Matricaria recutita L.)), cana-do-brejo (Costus spiralis (Jacq) Roscoe), chapéu-de-couro (Echinodorus macrophyllus (Cham. \& Schltdl.) Micheli), colônia (Alpinia zerumbet (Pers.) B.L.Burtt \& R.M.Sm.), confrei (Symphytum officinale L.), erva-cidreira (Lippia alba (Mill.) N.E.Br.), erva-debicho (Polygonum punctatum Elliot (sin: Polygonum acre H. B. K. var aquatile) / Polygon spectabilis L. / Polygonum hydropiperoides Michx.), espinheira-santa (Maytenus ilicifolia Mart. ex Reissek / Maytenus aquifolia Mart. / Sorocea bonplandii (Baill.) Burg. Lanj. \& Boer.), guaco (Mikania glomerata Sprengel), picão (Bidens pilosa L.), poejo (Mentha pulegium (Mill.) Lam. \& DC. ex. Briq.), sete-sangrias (Cuphea carthagenensis (Jacq.) J.F. Macbr.), sucupira (Pterodon emarginatus Vogel) e tanchagem (Plantago major L.). O abajerú, a erva de bicho e a espinheira-santa apresentam diferentes nomes científicos para um mesmo nome vulgar, que não constituem sinonímia (Jacomassi \& Machado, 2003; Azevedo \& Silva, 2006; MaioliAzevedo \& Fonseca-Kruel, 2007; Arjona et al., 2007; Silva \& Peixoto, 2009). Essas espécies são comercializadas como plantas medicinais com o mesmo nome popular, uma vez que apresentam morfologia semelhante e podem ou não conter as mesmas propriedades farmacológicas (Lorenzi \& Matos, 2002).

Apenas 11 das 20 plantas mais vendidas no Mercadão de Madureira estavam incluídas na listagem de plantas medicinais constantes na RDC-ANVISA no 10 (Anvisa, 2010a) (aroeira, boldo-do-Chile, camomila, chapéu-de-couro, erva-cidreira, erva-debicho, espinheira-santa, guaco, picão, poejo e tanchagem). Embora a arnica esteja incluída nessa resolução, a espécie Arnica montana nativa de

Rev. Bras. Pl. Med., Botucatu, v.14, n.3, p.537-547, 2012. 
regiões montanhosas da Europa não é a mesma da comercializada no Mercadão de Madureira (Solidago chilensis). No Brasil, a Solidago chilensis vem sendo utilizada em substituição a espécie européia, que não é cultivada no país (Lorenzi \& Matos, 2002).

A Relação Nacional de Plantas Medicinais de Interesse ao SUS é constituída de espécies vegetais cujo critério de inclusão tem por base 0 potencial de avançar nas etapas da cadeia produtiva e de gerar produtos de interesse ao SUS (Brasil, 2010). Essa relação contém 71 espécies (Brasil, 2009) e inclui 12 das 20 plantas mais vendidas no Mercadão de Madureira (aroeira, arruda, babosa, camomila, cana-do-brejo, colônia, erva-de-bicho, espinheira-santa, guaco, picão, poejo e tanchagem).

Cinco das espécies mais comercializadas no período estudado não estão contempladas por nenhuma das duas listagens (abajerú, arnica, confrei, sete-sangrias e sucupira).

O boldo (Plectranthus barbatus), amplamente cultivado em todo o Brasil, faz parte das duas listagens. Entretanto, a espécie comercializada no Mercadão de Madureira no período observado tratase do boldo-do-Chile (Peumus boldus).

Das 20 plantas medicinais mais comercializadas, seis apresentam propriedades tóxicas comprovadas dependendo do preparo e uso: arnica (Lameira \& Pinto, 2008; Saad et al., 2009), aroeira (Simões et al., 1999; Gomes et al., 2001; Veiga Junior \& Pinto, 2005), arruda (Gomes et al., 2001; Mengue et al., 2001; Ritter et al., 2002; Veiga Junior \& Pinto, 2005; Lameira \& Pinto, 2008), babosa (Gomes et al., 2001; Ritter et al., 2002; Veiga Junior \& Pinto, 2005; Castro, 2007; Negrelle et al., 2007; Lameira \& Pinto, 2008), confrei (Mengue et al., 2001; Gomes et al., 2001; Ritter et al., 2002; Veiga Junior \& Pinto, 2005; Castro, 2007; Negrelle et al., 2007; Lameira \& Pinto, 2008) e poejo (Mengue et al., 2001; Saad et al., 2009).

Foram obtidos, a partir da pesquisa bibliográfica, 118 artigos que contemplaram em seus títulos os nomes científicos de todas as 20 plantas selecionadas pelo estudo. As três plantas mais estudadas, presentes em $47,5 \%$ dos trabalhos nacionais pesquisados, foram a espinheira-santa, 0 guaco e a camomila, que constam tanto da listagem da RDC-ANVISA № 10, quanto da relação de interesse do SUS.

\section{Procedência e Coleta}

As plantas medicinais podem ser silvestres ou cultivadas. No município do Rio de Janeiro foi verificado relativo equilíbrio entre essas duas procedências (Azevedo \& Silva, 2006; Arjona et al., 2007). As plantas silvestres apresentam problemas na identificação das espécies, que só pode ser feita com segurança através da identificação botânica.
Muitas vezes até mesmo um botânico tem dificuldade em identificar uma planta que não se encontra no estágio de desenvolvimento que permita a diferenciação. A Farmacopéia Brasileira recebe permanentemente contribuições relacionadas à descrição de características morfoanatômicas que auxiliam na identificação das espécies, como a realizada por Leite et al. (2007) para o chapéu-decouro (Echinodorus macrophyllus). Na tradição brasileira, pessoas que se auto-intitulam "conhecedoras" selecionam espécies pretensamente fitoterápicas para serem utilizadas pela população (Wong \& Castro, 2003). Mengue et al. (2001) referemse a plantas morfologicamente semelhantes, mas com composição química bastante diversa, citando o caso de uma intoxicação causada por uma espécie de Digitalis identificada erroneamente como confrei (Symphytum oficinale L.). Barbosa et al. (2006) salientam que a qualidade dos fitoterápicos se inicia a partir da identificação correta da espécie.

A disponibilidade regional e sazonal das espécies favorece a ocorrência de falsificações, propiciando o comércio de uma espécie parecida na substituição de outra rara ou mesmo não disponível naquele local ou período do ano. Este é o caso da espinheira-santa (Maytenus spp.) (Almeida \& Albuquerque, 2002; Rodrigues \& Carlini, 2002), que devido ao excesso de exploração no Rio de Janeiro vem sendo substituída pela Sorocea bomplandii, sobre a qual não há estudos farmacológicos que comprovem o uso eficaz e seguro (Steenbock et al., 2003; Coulaud-Cunha et al., 2004; Ruschel \& Nodari, 2008; Silva \& Peixoto, 2009; Leitão et al., 2009). A fiscalização de órgãos ambientais tem impedido a extração de algumas espécies em áreas de proteção ambiental, fato este que pode estar estimulando a procura por espécies substitutas de aparência semelhante, caso do abajerú (Chrysobalanus icaco), cuja ação hipoglicemiante já está comprovada, que vem sendo substituído no Rio de Janeiro por uma espécie de outra família, Eugenia rotundifolia, que ainda não foi objeto de estudos farmacológicos (Silva \& Peixoto, 2009).

Pode ocorrer a contaminação de plantas medicinais por metais pesados oriundos do solo, da água e do ar (Tavares \& Carvalho, 1992; Capasso et al., 2000; Caldas \& Machado, 2004; Veiga Junior \& Pinto, 2005; Castro, 2007; Schwanz et al., 2008). O uso indevido de agrotóxicos no cultivo ou em plantações próximas contamina, não apenas com metais pesados, como também com organoclorados e organofosforados (Capasso et al., 2000; Zuin et al., 2003; Zuin et al., 2004;Castro, 2007). Por outro lado, Zaroni et al. (2004) afirmam que o cultivo orgânico, apesar de não deixar resíduos químicos prejudiciais à saúde, propiciam maior nível de contaminação microbiológica do que o cultivo tradicional. Estes 
autores associam a contaminação pelo microrganismo Escherichia coli com o preparo inadequado do adubo orgânico. Contudo, não descartam a possibilidade do solo ou mesmo da água utilizada para irrigação estarem contaminados com esse microrganismo.

As plantas silvestres também podem apresentar contaminação como é o caso das coletadas ao longo das rodovias (Schwanz et al., 2008; Dorigoni et al., 2001).

Tanto as plantas cultivadas como as silvestres sofrem grande variabilidade de princípios ativos. Estes são influenciados por diferentes fatores ambientais (localização geográfica, características do solo, clima) e genéticos (Wong \& Castro, 2003). A definição de um zoneamento agro-climático pode maximizar o rendimento dos princípios ativos a partir da seleção adequada do local de cultivo e da época de plantio (Marchese \& Figueira, 2005). Os estágios de desenvolvimento, as épocas do ano e os horários de coleta da planta também vão se refletir tanto nas propriedades medicinais quanto na toxicidade (Simões et al., 1999; Currier et al., 2000; Lima et al., 2001; Pimenta, 2002; Vargas, 2005; Marchese \& Figueira, 2005; Barbosa et al., 2006; Mariot \& Barbieri, 2007; Aguiar et al., 2008). Já a produtividade das plantas é influenciada pelo trato da cultura, em especial a irrigação e a adubação (Marchese \& Figueira, 2005). Pimenta (2002) constatou maior produção de princípios ativos no chapéu-de-couro (Echinodorus grandiflorus), cultivado sob luz plena na primavera. Esta espécie apresenta características e propriedades muito semelhantes à considerada nesse estudo, Echinodorus macrophyllus. Para a erva-cidreira (Lippia alba), Barros et al. (2009) obtiveram maior extração de óleos essenciais no verão e no outono.

Para algumas espécies, a decisão relativa à época do ano mais adequada para a colheita pode depender da concentração de princípio ativo que se deseja maximizar. A erva-de-bicho (Polygonum hydropiperoides), se coletada no outono, apresenta maior teor de polifenóis e tanino e, se coletada na primavera, maior teor de flavonóides (Jácome et al., 2004).

Capasso et al. (2000) preconizam que problemas de disponibilidade, adulteração, identificação botânica e flutuação da oferta podem ser minimizados quando se promove o cultivo em detrimento da extração silvestre. Além disso, afirmam que os cultivares de plantas medicinais propiciam o melhoramento genético, o elevado controle de qualidade e a manipulação agronômica. Zaroni et al. (2004) recomendam o treinamento dos agricultores por profissionais capacitados, uma vez que estes produzem a matéria-prima para a produção de medicamentos.

Quando a parte indicada como medicinal são as raízes e consequentemente, irão promover a retirada integral da planta, há uma vulnerabilidade das espécies silvestres, que correm o risco de ter a população reduzida ou até mesmo extinta em certas áreas (Pereira et al., 2007).

\section{Secagem e Armazenamento}

Zaroni et al. (2004) sugerem alguns cuidados a fim de reduzir a carga microbiana, como higiene adequada das mãos dos manipuladores das plantas medicinais; o material coletado deve ser colocado sobre superfície limpa; o recipiente de coleta deve ser limpo; deve-se procurar eliminar impurezas que possam acompanhar o órgão ou a planta recémcoletada; a secagem da planta deve ser realizada o mais rápido possível sem, entretanto, deixar de ser eficiente.

Alguns critérios devem ser respeitados na estocagem de plantas medicinais. O prédio deve ter piso de fácil limpeza, as embalagens devem ser mantidas acima do chão, a certa distância das paredes, em local seco, ventilado, protegido da luz, de insetos e de roedores. Além disso, para se evitar uma contaminação cruzada, deve-se manter separação física entre produtos diferentes (Marchese \& Figueira, 2005).

A observação do armazenamento e exposição das ervas nos boxes do Mercadão de Madureira permite afirmar que não há nenhum cuidado no manuseio que garanta a conservação e higiene. As ervas encontram-se dispostas sobre as bancadas dos boxes e são armazenadas diretamente no piso em ambiente escuro e úmido. São habitualmente embaladas em jornais reunindo molhos de diferentes espécies. Os molhos são amarrados com barbantes de algodão ou plástico em tamanhos não padronizados e ficam expostos a contaminação por insetos, aracnídeos e roedores. Os mesmos problemas foram encontrados por França et al. (2008), em Campina Grande na Paraíba.

A preservação dos princípios ativos pode ser comprometida por secagem e armazenamento inadequado, pois pode ocorrer degradação por processos metabólicos, hidrólise, luz, ação enzimática, oxidação, fermentação, calor e contaminação microbiológica (Costa et al., 1999; Vargas, 2005; Veiga Junior \& Pinto, 2005; Marchese \& Figueira, 2005; Barbosa et al., 2006; Araújo et al., 2009).

Barbosa et al. (2006) afirmam que cada espécie de planta deve ter o próprio processo de secagem, pois apresenta comportamento diferenciado em relação à preservação dos princípios ativos para mesma temperatura do ar. Recomendam, por exemplo, que a secagem de erva-cidreira (Lippia alba) para fins de comercialização deve ser realizada utilizando ar aquecido entre 40 e $80^{\circ} \mathrm{C}$ para preservação do teor adequado do óleo essencial. 
Costa et al.(1999) verificaram que a secagem sem prévia lavagem de folhas de guaco (Mikania glomerata) resultou em maior contaminação microbiológica. Lameira \& Pinto (2008) constataram que estas quando mofadas podem impedir a coagulação do sangue. O excesso de calor na secagem é prejudicial, pois muitas substâncias químicas, tais como os óleos essenciais, volatilizam mesmo a temperatura ambiente (Brandão et al., 2002).

O tempo de armazenamento pode acarretar mudanças na qualidade e/ou na quantidade das substâncias ativas das plantas (Simões et al., 1999).

Após a secagem deve-se observar o acondicionamento em embalagem apropriada, que evite o risco de ataque de pragas de armazenamento e a absorção de umidade do ambiente (Marchese \& Figueira, 2005). Em estudo realizado em Mato Grosso do Sul a maioria das amostras de plantas medicinais comercializadas, incluindo o chapéu-de-couro (Echidodorus macrophyllos), apresentava sujidades, presença de insetos e contaminação por fungos (Nunes et al., 2003). A presença de flores de espécies estranhas e de insetos foi observada em grupos de amostras de camomila (Chamomilla recutita) (Brandão et al., 1998; Brandão et al., 2002). O boldo-do-Chile (Peumus boldus), comercializado em mercados e ervanários, apresentou contaminação fúngica incluindo fungos toxicogênicos em estudos realizados nas cidades de Curitiba (Barbosa et al., 2001), São Luís (Amaral et al., 2003) e Campinas (Rocha et al., 2004). Em Recife, estudo no comércio de ervas em farmácias detectou matérias estranhas acima do permitido em 2/3 das amostras (Nascimento et al., 2005). Em São Luís, estudo realizado em cinco mercados públicos verificou que a maioria das amostras de plantas para uso medicinal estava imprópria para consumo devido à elevada contaminação por material estranho, sendo que nas amostras de folhas de boldo-do-Chile (Peumus boldus) constatou-se 18 a $20 \%$ de partes diferentes do mesmo vegetal e/ou outro vegetal, indicativo de contaminação ou fraude (Amaral et al., 2003). Um raizero de Diadema relatou um caso de intoxicação ao vender um pacote de chá contaminado por fungos, devido ao mau processo de secagem e acondicionamento das plantas (Lanini et al., 2009).

\section{Comércio}

Coulaud-Cunha et al. (2004) verificaram que a maioria dos comerciantes dos mercados e feiras populares do município do Rio de Janeiro não se enquadra no conceito de erveiro ("pessoas detentoras de conhecimento popular sobre plantas medicinais, as vezes passada de geração para geração", p.51). Os vendedores apenas compram ervas na central de abastecimento e as revendem, se tornando os responsáveis pela sua dispensação. As orientações de indicação e uso são repassadas para o comprador a partir de informações obtidas com o fornecedor ou em revistas de leitura popular.

A influência da imprensa na difusão de informações errôneas sobre os efeitos das plantas medicinais é muito grande e, além disso, sem qualquer controle na maioria dos países. No Brasil é comum ouvir em propagandas a expressão: "não faz mal para a saúde porque é $100 \%$ natural" (Veiga Junior \& Pinto, 2005; Azevedo \& Silva, 2006). Os meios de comunicação influenciam tanto na demanda da população por espécies, como também nas indicações terapêuticas prestadas pelos comerciantes de plantas medicinais (Nunes et al., 2003; Azevedo \& Silva, 2006; Maioli-Azevedo et al., 2007).

$\mathrm{Na}$ comercialização popular de plantas medicinais pode ocorrer dificuldade por parte do comerciante, fornecedor e consumidor em identificar corretamente uma planta. Plantas diferentes conhecidas pelo mesmo nome popular são comercializadas sem que haja muitas vezes a comprovação de suas propriedades farmacológicas e segurança toxicológica. Esse é o caso da espinheira-santa (Maytenus ilicifolia / Maytenus aquifolium / Sorocea bonplandii), do abajerú (Chrysobalanus icaco / Eugenia rotundifolia), do boldo (Peumus boldus / Plectranthus barbatus), da ervade-bicho (Polygonum punctatum / Polygon spectabilis / Polygonum hydropiperoides) e da arnica (Solidago chilensis / Arnica montana). Mesmo os órgãos fiscalizadores podem ter dificuldade em diferenciar espécies morfologicamente semelhantes, uma vez que apenas partes das plantas são comercializadas, geralmente secas, o que impossibilita até mesmo a identificação botânica (Coulaud-Cunha et al., 2005; Ruschel \& Nodari, 2008; Silva \& Peixoto, 2009).

As possibilidades de adulteração são elevadas mesmo em se tratando do comércio de plantas medicinais em farmácias e lojas de produtos naturais. A rotulação industrial nas preparações vegetais não constitui uma garantia (Veiga Junior \& Pinto, 2005).

O controle da comercialização de plantas medicinais pelos órgãos oficiais em feiras livres, mercados públicos ou lojas de produtos naturais ainda é insipiente (Silva \& Peixoto, 2009).

\section{Modo de preparo pelo usuário}

A preparação e o armazenamento inadequados do preparado contribuem para o risco de toxicidade (Capasso et al., 2000), podendo ocasionar intoxicações agudas ou crônicas (Rocha et al., 2004), bem como variações da concentração dos princípios ativos (Lameira \& Pinto, 2008).

A parte da planta a ser utilizada em cada preparado deve ser cuidadosamente observada, uma 
vez que contém diferentes princípios ativos que apresentam propriedades terapêuticas ou tóxicas. Há plantas cuja toxicidade já está bem estudada tais como a babosa, cuja ação nefrotóxica é conhecida (Morais et al., 2005), e a tanchagem, cuja casca da semente nunca deve ser utilizada (Anvisa, 2010a). Nesses casos deve ser estabelecido um mecanismo de transmissão de informação ao usuário, para minimizar os riscos de efeitos indesejáveis.

Um estudo etnobotânico ressalta a importância do preparo de chás ser realizado sem submeter a planta à fervura, demonstrando preocupação com a possível perda de princípios ativos no processo de decocção (Baldauf et al., 2009). Por outro lado, diversos contaminantes presentes na planta não são eliminados mesmo no processo de infusão. O emprego da fervura também não garante a segurança do preparado, como no caso relatado por Rocha et al. (2004) em amostras de boldo-do-Chile (Peumus boldus) contaminadas por Aspergillus (ponto de fusão em torno de $269^{\circ} \mathrm{C}$ ), que se manteve potencialmente carcinogênico. A decisão pelo uso de folhas frescas ou secas, também pode alterar a concentração de princípios ativos. Raizeiros de Diadema relatam o aparecimento de sintomas desagradáveis no uso de folhas frescas, que foram por eles consideradas potencialmente tóxicas por apresentarem maior concentração de princípios ativos (Lanini et al., 2009).

\section{Uso da planta medicinal}

Poucas plantas medicinais foram submetidas a estudos pré-clínicos e clínicos a fim de comprovar sua eficácia e segurança (Capasso et al., 2000; Turolla \& Nascimento, 2006; Silveira et al., 2008; Silva \& Peixoto, 2009). No Brasil, a pesquisa com plantas nativas sofre da carência de recursos e de dificuldades legais e burocráticas para o acesso, coleta de plantas e o desenvolvimento de estudos (Carlini et al., 2007).

Muitas plantas medicinais amplamente utilizadas na cultura popular não possuem seu perfil tóxico bem conhecido. O chapéu-de-couro (Echinodorus macrophyllus), por exemplo, teve o primeiro estudo hematológico realizado por Moreti et al. (2006), que revelou alterações nas séries branca e vermelha de cobaias. A atividade citotóxica da colônia (Alpinia zerumbet) foi estudada em apenas dois trabalhos, se mostrando ausente (Correa et al., 2010).

O fato de bula não acompanhar cada planta medicinal implica em que a orientação para o uso seja baseada nas informações prestadas pelos erveiros, por alguém de seu relacionamento ou pela própria experiência. Um estudo etnobotânico aponta que a dosagem e o tempo de tratamento não estão previamente estabelecidos pela população, que utiliza plantas medicinais até a cura ou em função de critérios subjetivos (Pereira et al., 2005).
Plantas como a aroeira, arnica, arruda, babosa, confrei e o poejo, que apresentam alguma toxicidade já reconhecida cientificamente podem ser objeto de erros de administração e/ou uso indevido.

O potencial tóxico teratogênico e abortivo de algumas espécies vegetais incluindo a arruda, babosa, boldo-do-Chile, camomila, confrei, espinheirasanta, guaco, tanchagem, foram reconhecidos e contra-indicados para gestantes e/ou lactantes no Estado do Rio de Janeiro (Rio de Janeiro, 2002). O boldo-do-Chile (Peumus boldus) também apresenta indícios de hepatotoxicidade, devendo ser consumido com moderação (Ruiz et al., 2008).

Oito das plantas estudadas (boldo-do-Chile, chapéu-de-couro, erva-cidreira, erva-de-bicho, espinheira-santa, picão, poejo e tanchagem) apresentam contra-indicações listadas na RDCANVISA no 10 (Anvisa, 2010a) para o uso em algum período da vida, como gravidez e infância ou na presença de doenças pré-existentes, crônicas ou não.

Pacientes costumam não informar os médicos que estão fazendo uso de plantas medicinais. Esse procedimento pode contribuir para a ocorrência das interações medicamentosas (Capasso et al., 2000; Wong \& Castro, 2003; Cordeiro et al., 2005; Silveira et al., 2008).

\section{Auto Diagnóstico / Automedicação}

$\mathrm{O}$ auto diagnóstico antecede a busca pelo tratamento a base de plantas medicinais. O erveiro parte de um diagnóstico pré-estabelecido pelo consumidor para recomendar a planta mais adequada aos agravos apontados.

Os fitoterápicos e as plantas medicinais estão diretamente ligados à automedicação (Rates, 2001). O uso se baseia na orientação farmacêutica e indicação leiga, respectivamente. Mesmo a indicação farmacêutica fornecida ao paciente é de baixa qualidade já que essa utiliza como fonte principal, a literatura promocional dos laboratórios fabricantes (Rates, 2001).

No caso de tratamento de doenças autolimitadas, o uso de plantas medicinais tem o mesmo papel dos medicamentos de venda livre. No entanto, no caso de doenças infecciosas, metabólicas e outras que requerem acompanhamento médico, o uso das plantas medicinais pode retardar o diagnóstico e o tratamento adequados. Há também o risco do agravamento do estado de saúde do usuário (Ritter et al., 2002; Wong \& Castro, 2003).

Plantas medicinais podem ser também potencialmente prejudiciais ao atrasar ou substituir um tratamento convencional ou quando comprometem a eficácia de medicamentos convencionais (Capasso et al., 2000; Wong \& Castro, 2003). Estudo em hospital infantil em João Pessoa mostra que quase $1 / 3$ dos responsáveis pelas crianças internadas

Rev. Bras. PI. Med., Botucatu, v.14, n.3, p.537-547, 2012. 
administraram plantas medicinais antes de procurar o serviço hospitalar e, mais de $40 \%$ associaram plantas medicinais a medicamentos (Tôrres et al., 2005).

Estudo de Negrelle et al. (2007) com 50 famílias entrevistadas mostra que nenhuma utilizou plantas medicinais sob orientação médica e que $80 \%$ dessas apresentavam algum registro de toxicidade e contra-indicações na literatura.

\section{DISCUSSÃO}

Apesar do presente estudo se restringir as 20 espécies mais comercializadas em agosto de 2008 no Mercadão de Madureira, os resultados observados podem ser generalizados.

Embora haja clara distinção entre plantas medicinais e fitoterápicos explicitada na legislação, a abordagem reguladora trata basicamente dos fitoterápicos. As plantas são consideradas como matéria-prima e não como produtos. Na prática, elas podem ser comercializadas e consumidas diretamente pela população, sem estarem sujeitas a qualquer controle, como pode ser observado no Mercadão de Madureira.

Dentre as plantas estudadas, as espécies babosa, boldo-do-Chile, camomila, confrei, espinheira-santa, e guaco constam da lista de medicamentos fitoterápicos de registro simplificado, e, por essa razão, não necessitam validar as indicações terapêuticas e segurança de uso (Anvisa, 2008). Já para o consumo direto dessas plantas pela população todos os problemas associados ao processo produtivo estão presentes.

O regulamento próprio para plantas medicinais in natura e drogas vegetais manipuladas não está ainda estabelecido. Como consequência, o comércio de plantas embaladas e rotuladas ainda é bastante precário. A iniciativa da ANVISA (Anvisa, 2010a) em publicar informações sobre posologia, modo de usar, via, indicações de uso, contraindicações e efeitos adversos de 66 espécies de plantas medicinais, está restrita a drogas vegetais, ou seja, não se aplica diretamente aos consumidores de plantas in natura das feiras e mercados populares.

A União Européia mantém um guia de práticas agrícolas para plantas medicinais e aromáticas, que prevê cuidados desde a semeadura, cultivo, colheita, processamento, empacotamento, estocagem e transporte de todo o material envolvido na produção de plantas medicinais (Marchese \& Figueira, 2005). O estudo do processo produtivo desenvolvido no presente trabalho aponta para a pertinência de um guia como esse, uma vez que todas as etapas consideradas apresentaram citação de problemas na literatura.

Cinco das plantas mais comercializadas no Mercadão de Madureira (abajerú, confrei, sucupira, sete-sangrias e arnica) não constam da relação de plantas de interesse do SUS (Brasil, 2010), nem da lista de plantas para a produção de drogas vegetais (Anvisa, 2010a). Se, por um lado, a presença de uma planta nessas listagens não garante o crescimento das pesquisas científicas, por outro, a ausência desestimula a seleção para estudos. Plantas, como o confrei, podem não estar presentes pela já estabelecida toxicidade para uso interno. No caso da arnica, o fato de existirem duas espécies comercializadas com o mesmo nome popular, não dispensa a necessidade de estudos específicos para a espécie nativa brasileira.

Diversas plantas medicinais já contam com muitos estudos abordando, tanto as propriedades farmacológicas, quanto os efeitos tóxicos. Não obstante, mesmo as plantas mais estudadas, podem apresentar ainda carência de investigações em aspectos específicos. Correa et al. (2010) consideram que as revisões bibliográficas são importantes instrumentos não só para dar embasamento teórico e metodológico, mas também para apontar as áreas de conhecimento que ainda não foram exploradas por outros pesquisadores, facilitando assim a seleção de estudos futuros.

\section{REFERÊNCIA}

AGUIAR, J.S. et al. Atividade antimicrobiana de Lippia alba (Mill.) N.E. Brown (Verbenaceae). Revista Brasileira de Farmacognosia, v.18, n.3, p.436-40, 2008.

ALMEIDA, C.F.C.B.R.; ALBUQUERQUE, U.P. Uso e conservação de plantas e animais medicinais no estado de Pernambuco (Nordeste do Brasil): um estudo de caso. Interciência, v.27, n.6, p.276-85, 2002.

AMARAL, F.M.M. et al. Avaliação da qualidade de drogas vegetais comercializadas em São Luís/Maranhão. Revista Brasileira de Farmacognosia, v.13, supl., p.2730, 2003.

ANVISA. Instrução Normativa no 5, de 11 de dezembro de 2008. Determina a publicação da "Lista de medicamentos fitoterápicos de registro simplificado". Diário Oficial da União, № 242, 12 de dezembro de 2008, p. 56. Disponível em: <ftp://ftp.saude.sp.gov.br/ftpsessp/ bibliote/informe_eletronico/2008/iels.dez.08/iels236/ U_IN-ANVISA-5_111208.pdf>. Acesso em: 08 mar.2010. ANVISA. Resolução da Diretoria Colegiada (RDC) no 10, de 9 de março de 2010(a). Dispõe sobre a notificação de drogas vegetais junto à Agência Nacional de Vigilância Sanitária (ANVISA) e dá outras providências. Diário Oficial da União, № 46, 10 de março de 2010. ANVISA. Resolução da Diretoria Colegiada (RDC) no 14 , de 31 de março de 2010(b). Dispõe sobre o registro de medicamentos fitoterápicos. Diário Oficial da União, № 63, 5 de abril de 2010.

ARAÚJO, A.C. et al. Caracterização sócio-econômicocultural de raizeros e procedimentos pós-colheita de 
plantas medicinais comercializadas em Maceió, AL. Revista Brasileira de Plantas Medicinais, v.11, n.1, p.8191, 2009.

ARJONA, F.B.S.; MONTEZUMA, R.C.; SILVA, I.M. Aspectos etnobotânicos e biografia de espécies medicinais e/ou rituais comercializadas no mercado de Madureira, RJ. Caminhos de Geografia, v.8, n.23, p.41-50, 2007. Disponível em: <http://www.ig.ufu.br/revista/caminhos. html>. Acesso: em 08 mar.2010.

AZEVEDO, S.K.; SILVA, I.M. Plantas medicinais e de uso religioso comercializadas em mercados e feiras livres no Rio de Janeiro, RJ, Brasil. Acta Botanica Brasilica, v.20, n.1, p.185-94, 2006.

BALDAUF, C. et al. "Ferveu, queimou o ser da erva": conhecimentos de especialistas locais sobre plantas medicinais na região Sul do Brasil. Revista Brasileira de Plantas Medicinais, v.11, n.3, p.282-91, 2009.

BARBOSA, M.C.S. et al. Avaliação da qualidade de folhas de boldo-do-chile (Peumus boldus Molina) comercializadas em Curitiba, PR. Revista Brasileira de Farmacognosia, v.11, n.1, p.1-4, 2001.

BARBOSA, F.F. et al. Influência da temperatura do ar de secagem sobre o teor e a composição química do óleo essencial de Lippia alba (Mill.) N.E. Brown. Química Nova, v.29, n.6, p.1221-5, 2006.

BARROS, F.M.C. et al. Variabilidade sazonal e biossíntese de terpenóides presentes no óleo essencial de Lippia alba (Mill.) N.E. Brown (Verbenaceae). Química Nova, v.32, n.4, p.861-7, 2009.

BRANDÃO, M.G.L.; FREIRE, N.; VIANNA-SOARES, C.D. Vigilância de fitoterápicos em Minas Gerais. Verificação da qualidade de diferentes amostras comerciais de camomila. Cadernos de Saúde Pública, v.14, n.3, p.6136, 1998.

BRANDÃO, M.G.L. et al. Qualidade de amostras comerciais de chás de plantas medicinais. Revista Brasileira de Plantas Medicinais, v.5, n.1, p.56-9, 2002. BRASIL. Ministério da Saúde, Secretaria de Ciência, Tecnologia e Insumos Estratégicos, Departamento de Assistência Farmacêutica. A fitoterapia no SUS e o Programa de Pesquisa de Plantas Medicinais da Central de Medicamentos. Brasília: Ministério da Saúde, 2006. 148p.

BRASIL. Ministério da Saúde. RENISUS - Relação Nacional de Plantas Medicinais de Interesse ao SUS. Espécies vegetais. DAF/SCTIE/MS - RENISUS - fev/2009. Disponível em: <http://portal.saude.gov.br/portal/ arquivos/pdf/RENISUS.pdf>. Acesso: em 20 jul.2010.

BRASIL. Ministério da Saúde. Sobre a RENISUS. Disponível em: <http://portal.saude.gov.br/ portal/saude/ profissional/visualizar_texto.cfm?idtxt=30780>. Acesso em: 20 jul.2010.

CALDAS, E.D.; MACHADO, L.L. Cadmium, mercury and lead in medicinal herbs in Brazil. Foods and Chemical Toxicology, v.42, p.599-603, 2004.

CALIXTO, J.B. Efficacy, safety, quality control, marketing and regulatory guidelines for herbal medicines (Phytotherapeutic agents). Brazilian Journal of Medical and Biological Research, v.33, n.1, p.179-89, 2000.

CAPASSO, R. et al. Phytotherapy and quality of herbal medicines. Fitoterapia, v.71, p.S58-S65, 2000.

CARLINI, E.A. et al. Da planta medicinal ao medicamento. Scientific American Brasil, v.63, p.70-7, 2007.
CASTRO, E.G.R. Aspectos toxicológicos e interações medicamentosas dos fitoterápicos. In: LOPES, C.A. (Ed.). Diagnóstico e Tratamento. v.3. São Paulo: Manole, 2007, p.764-77.

CORDEIRO, C.H.G.; CHUNG, M.C.; SACRAMENTO, L.V.S. Interações medicamentosas de fitoterápicos e fármacos: Hypericum perforatum e Piper methysticum. Revista Brasileira de Farmacognosia, v.15, n.3, p.272-8, 2005. CORREAA, M.P. Dicionário de plantas úteis do Brasil e das exóticas cultivadas. Rio de Janeiro: Imprensa Nacional, 1984.

CORREA, A.J.C.; LIMA, C.E.; COSTA, M.C.C.D. Alpinia zerumbet (Pers.) B.L.Burtt \& R.M.Sm. (Zingiberaceae): levantamento de publicações nas áreas farmacológicas e química para o período 1987-2008. Revista Brasileira de Plantas Medicinais, v.12, n.1, p.113-9, 2010.

COSTA, C.C.; CASALI, V.W.D.; MACEDO, J.A.B. Qualidade de folhas de Guaco (Mikania glomerata Sprengel) após secagem e embalagem. Revista Brasileira de Plantas Medicinais, v.1, n.2, p.1-5, 1999.

COULAUD-CUNHA, S.; OLIVEIRA, R.S.; WAISSMANN, W. Venda livre de Sorocea bomplandii Bailon como Espinheira Santa no município do Rio de Janeiro - RJ. Revista Brasileira de Farmacognosia, v.14, supl. 1, p.513, 2004.

CURRIER, S.J.; JOHNSTON, P.D.; GORELICK, K.J. Herbal medicines. Science and Medicine, v.7,n.1, p.403, 2000.

DORIGONI, P.A. et al. Levantamento de dados sobre plantas medicinais de uso popular no município de São João do Polêsine, RS, Brasil. I - Relação entre enfermidades e espécies utilizadas. Revista Brasileira de Plantas Medicinais, v.4, n.1, p.69-79, 2001.

FONTE, N.N.; CAVALLET, V.J.; BIASI, L.A. A complexidade do trabalho com plantas medicinais: uma reflexão necessária. Revista Brasileira de Plantas Medicinais, v.7, n.1, p.16-22, 2004.

FRANÇA, I.S.X. et al. Medicina popular: benefícios e malefícios das plantas medicinais. Revista Brasileira de Enfermagem, v.61, n.2, p.201-8, 2008.

GOMES, E.C. et al. Plantas medicinais com características tóxicas usadas pela população do município de Morretes, PR. Revista Visão Acadêmica, v.2, n.2, p.7780, 2001.

JACOMASSI, E.; MACHADO, S.R. Características anatômicas da espinheira-santa (Maytenus ilicifolia Mart. ex Reissek e Maytenus aquifolia Mart.) e mata-olho (Sorocea bonplandii (Baill.) Burg. Lanj. \& Boer.) para o controle de qualidade da matéria prima. Revista Brasileira de Plantas Medicinais, v.6, n.1, p.84-96, 2003. JÁCOME, R.L.R.P. et al. Caracterização farmacognóstica de Polygonum hydropiperoides Michaux e P. spectabile (Mart.) (Polygonaceae). Revista Brasileira de Farmacognosia, v.14, n.1, p.21-7, 2004.

LAMEIRA, O.A.; PINTO, J.E.B.P. (Eds). Plantas medicinais: do cultivo, manipulação e uso à recomendação popular. Belém: Embrapa Amazônia Oriental, 2008. 264p.

LANINI, J. et al. "O que vêm da terra não faz mal" - relatos de problemas relacionados ao uso de plantas medicinais por raizeiros de Diadema/SP. Revista Brasileira de Farmacognosia, v.19, n.1A, p.121-9, 2009. LEITÃO, F. et al. Urban ethnobotany in Petrópolis and Nova Friburgo (Rio de Janeiro, Brazil). Revista Brasileira

Rev. Bras. PI. Med., Botucatu, v.14, n.3, p.537-547, 2012. 
de Farmacognosia, v.19, n.1B, p.333-42, 2009.

LEITE, J.P.V. et al. Contribuição ao estudo farmacobotânico da Echinodorus macrophyllus (Kunth) Micheli (chapéu de couro) - Alismataceae. Revista Brasileira de Farmacognosia, v.17, n.2, p.242-8, 2007.

LIMA, S.S. et al. Cultura monocional de erva de bicho (Polygonum acre H. B. K. var aquatile) para produção de um fitoterápico em escala comercial. Revista Brasileira de Plantas Medicinais, v.4, n.1, p.51-5, 2001.

LORENZI, H.; MATOS, F.J.A. Plantas medicinais no Brasil: nativas e exóticas cultivadas. Nova Odessa, SP: Instituto Plantarum, 2002. 544p.

MAIOLI-AZEVEDO, V.; FONSECA-KRUEL, V.S. Plantas medicinais e ritualísticas vendidas em feiras livres do município do Rio de Janeiro, RJ, Brasil: estudo de caso nas zonas Norte e Sul. Acta Botânica Brasílica, v.21, n.2, p.263-75, 2007.

MARIOT, M.P.; BARBIERI, R.L. O conhecimento popular associado ao uso da espinheira-santa (Maytenus ilicifolia e M. aquifolium). Revista Brasileira de Biociências, v.5, supl.1, p.666-8, 2007.

MARIOT, M.P.; BARBIERI, R.L. Metabólitos secundários e propriedades medicinais da espinheira-santa (Maytenus ilicifolia Mart. Ex Reiss. e M. aquifolium Mart.). Revista Brasileira de Plantas Medicinais, v.9, n.3, p.8999, 2007.

MARCHESE, J.A.; FIGUEIRA, G.M. O uso de tecnologias pré e pós-colheita e boas práticas agrícolas na produção de plantas medicinais e aromáticas. Revista Brasileira de Plantas Medicinais, v.7, n.3, p.86-96, 2005.

MENGUE, S.S.; MENTZ, L.A.; SCHENCKEL, E.P. Uso de plantas medicinais na gravidez. Revista Brasileira de Farmacognosia, v.11, n.1, p.21-35, 2001.

MORAIS, S.M. et al. Plantas medicinais usadas pelos índios Tapebas do Ceará. Revista Brasileira de Farmacognosia, v.15, n.2, p.169-77, 2005.

MORETI, D.L.C. et al. Estudo hematológico em ratos sob a ação de plantas medicinais. XXXVII. Echinodorus macrophylus (Kunth.) Mitch. Revista Brasileira de Plantas Medicinais, v.8, n.4, p.162-4, 2006.

NASCIMENTO, V.T. et al. Controle de qualidade de produtos à base de plantas medicinais comercializados na cidade de Recife - PE: erva-doce (Pimpinella anisum L.), quebra-pedra (Phyllanthus spp.), espinheira-santa (Maytenus ilicifolia Mart.) e camomila (Matricaria recutita L.). Revista Brasileira de Plantas Medicinais, v.7, n.3, p.56-64, 2005.

NEGRELLE, R.R.B. et al. Estudo etnobotânico junto a Unidade Saúde da Família Nossa Senhora dos Navegantes: subsídios para o estabelecimento de programa de fitoterápicos na Rede Básica de Saúde do município de Cascavel (Paraná). Revista Brasileira de Plantas Medicinais, v.9, n.3, p.6-22, 2007.

NUNES, G.P. et al. Plantas medicinais comercializadas por raizeiros no Centro de Campo Grande, Mato Grosso do Sul. Revista Brasileira de Farmacognosia, v.13, n.2, p.83-92, 2003.

PEREIRA, C.O. et al. Abordagem etnobotânica de plantas medicinais utilizadas em dermatologia na cidade de João Pessoa-Paraíba. Revista Brasileira de Plantas Medicinais, v.7, n.3, p.9-17, 2005.

PEREIRA, Z.V. et al. Levantamento das plantas medicinais do cerrado Sensu Stricto da fazenda Paraíso
- Dourado, MS. Revista Brasileira de Biociências, v.5, supl. 1, p.249-51, 2007.

PIMENTA, D.S. Contribuição a ecologia, cultivo e validação do uso de Echinodorus grandiflorus (Chamisso \& Schlechtendal) Micheli (Chapéu-decouro). 2002. 179p. Tese (Doutorado - Área de Concentração em Biologia Celular e Molecular) - Instituto Oswaldo Cruz, Fundação Oswaldo Cruz, Rio de Janeiro. RATES, S.M.K. Promoção do uso racional de fitoterápicos: uma abordagem no ensino de Farmacognosia. Revista Brasileira de Farmacognosia, v.11, n.2, p.57-69, 2001.

RIO DE JANEIRO. SECRETARIA DE ESTADO DE SAÚDE. Resolução SES/RJ № 1757, de 18 de fevereiro de 2002. Contra-indica o uso de plantas medicinais no âmbito do Estado do Rio de Janeiro e dá outras providências. Diário Oficial do Estado do Rio de Janeiro, 20 de fevereiro de 2002.

RITTER, M.R. et al. Plantas usadas como medicinais no município de Ipê, RS, Brasil. Revista Brasileira de Farmacognosia, v.12, n.2, p.51-62, 2002.

ROCHA, L.O.; SOARES, M.M.S.R.; CORRÊA, C.L. Análise da contaminação fúngica em amostras de Cássia acutifólia Delile (sene) e Peumus boldus (Molina) Lyons (boldo-do-Chile) comercializadas na cidade de Campinas, Brasil. Revista Brasileira de Ciências Farmacêuticas v.40, n.4, p.521-7, 2004.

RODRIGUES, E.; CARLINI, E.A. A importância dos levantamentos etnofarmacológicos no desenvolvimento de fitomedicamentos. Revista Racine, v.70, p.30-5, 2002. RUIZ, A.L.T.G. et al. Farmacologia e toxicologia de Peumus boldus e Baccharis genistelloides. Revista Brasileira de Farmacognosia, v.18, n.2, p.295-300, 2008. RUSCHEL, A.R.; NODARI, R.O. Colheita foliar da cancorosa [Sorocea bonplandii (Baill.) Burg., Lanj. \& W. Boer]: uma espinheira-santa da Mata Atlântica. Revista Brasileira de Plantas Medicinais, v.10, n. 4, p.43-50, 2008.

SAAD, G.A. et al. Fitoterapia contemporânea: tradição e ciência na prática clínica. Rio de Janeiro: Elsevier, 2009, 402p.

SCHWANZ, M. et al. Análise de metais pesados em amostras de Peumus boldus Mol. (Monimiaceae). Revista Brasileira de Farmacognosia, v.18, n.1, p.98101, 2008.

SILVA, I.M.; PEIXOTO, A.L. O abajurú (Chrysobalanus icaco L. e Eugenia rotundifolia Casar. comercializado na cidade do Rio de Janeiro, Brasil. Revista Brasileira de Farmacognosia, v.19, n.1B, p.325-32, 2009.

SILVEIRA, P.F.; BANDEIRA, M.A.M.; ARRAIS, P.S.D. Farmacovigilância e reações adversas às plantas medicinais e fitoterápicos: uma realidade. Revista Brasileira de Farmacognosia, v.18, n.4, p.618-26, 2008. SIMÕES, C.M.O. et al. Farmacognosia: da planta ao medicamento. 1.ed. Porto Alegre/Florianópolis: Editora da UFRGS/Editora da UFSC, 1999. 821p.

SINITOX (Sistema Nacional de Informações TóxicoFarmacológicas), Instituto de Comunicação e Informação Científica e Tecnológica em Saúde, Fundação Oswaldo Cruz. Estatística anual de casos de intoxicação e envenenamento. Disponível em: <www.sinitox.icict. fiocruz.br>. Acesso em: 29 jun.2010.

STEENBOCK, W. et al. Avaliação de características

Rev. Bras. PI. Med., Botucatu, v.14, n.3, p.537-547, 2012. 
fenotípicas para determinação do rendimento foliar em espinheira-santa (Maytenus ilicifolia Martius). Revista Brasileira de Plantas Medicinais, v.6, n.1, p.71-6, 2003.

TAVARES, T.M.; CARVALHO, F.M. Avaliação de exposição de populações humanas a metais pesados no ambiente: exemplos do recôncavo baiano. Química Nova, v.15, n.2, p.147-54, 1992.

TÔRRES, A.R. et al. Estudo sobre o uso de plantas medicinais em crianças hospitalizas da cidade de João Pessoa: riscos e benefícios. Revista Brasileira de Farmacognosia, v.15, n.4, p.373-80, 2005.

TUROLLA, M.S.R.; NASCIMENTO, E.S. Informações toxicológicas de alguns fitoterápicos utilizados no Brasil. Revista Brasileira de Ciências Farmacêuticas, v.42, n.2, p.289-306, 2006.

VARGAS, C.E. Extração supercrítica do óleo essencial do abajeru (Chrysobalanus icaco). 2005, 93p. Dissertação (Mestrado em Engenharia Química) Coordenação dos Programas de Pós Graduação de Engenharia, Universidade Federal do Rio de Janeiro,
Rio de Janeiro.

VEIGA JUNIOR, V.F.; PINTO, A.C.; MACIEL, A.M. Plantas medicinais: cura segura? Química Nova, v.28, n.3, p.519-28, 2005.

ZARONI, M. et al. Qualidade microbiológica das plantas medicinais produzidas no Estado do Paraná. Revista Brasileira de Farmacognosia, v.14, n.1, p.29-39, 2004. ZUIN, V.G.; YARIWAKE, J.H.; BICCHI, C. Fast supercritical fluid extraction and high-resolution gas chromatography with electron-capture and flame photometric detection for multiresidue screening of organochlorine and organophosphorus pesticides in Brazil's medicinal plants. Journal of Chromatography A, v.985, p.59-166, 2003.

ZUIN, V.G.; YARIWAKE, J.H.; BICCHI, C. Avaliação da qualidade de drogas vegetais à base de Passiflora spp. comercializadas no Brasil: presença de resíduos de pesticidas. Revista Brasileira de Plantas Medicinais, v.6, n.2, p.60-6, 2004.

WONG, A.; CASTRO E.G.R. Aspectos toxicológicos dos fitoterápicos. Arquivos Brasileiros de Fitomedicina Científica, v.1, n.2, p.96-102, 2003. 